\title{
Graft Copolymerization of Polydimethylsiloxane Macromonomers with Isopropenyl-1,3,5-triazines
}

\author{
Keiji Maeda, Yasuo Yuki, Hideo Kunisada, \\ Shuji Kondo, and Kazuyasu TANAKA \\ Department of Materials Science and Engineering, \\ Nagoya Institute of Technology, Gokiso-cho, \\ Showa-ku, Nagoya 466, Japan
}

(Received March 23, 1992)

\begin{abstract}
Polydimethylsiloxane macromonomers $\left(\mathrm{M}_{1}\right)$ having various molecular weights were copolymerized with two isopropenyltriazines $\left(\mathrm{M}_{2}\right)$, 2-amino-4-anilino- and 4- $N$-octadecylanilino)-6-isopropenyl-1,3,5-triazine (AAIT and N18), using azobisisobutyronitrile as an initiator in tetrahydrofuran. $r_{1}$ decreased from 0.50 to 0.10 and $r_{2}$ increased from 1.97 to 6.47 with the molecular weight of macromonomer for AAIT $\left(\mathrm{M}_{2}\right)$. Glass transition temperature $\left(T_{\mathrm{g}}\right)$ of the graft copolymers consisting of AAIT segments slightly increased with the molecular weight of macromonomer. The second $T_{\mathrm{g}}$ of silicone segment was observed the below $-100^{\circ} \mathrm{C}$. The degree of lowering of $T_{\mathrm{g}}$ for the graft copolymers of N18 was larger than that of graft copolymers of AAIT with increasing the siloxane content.

KEY WORDS Macromonomer / Graft Copolymer / Isopropenyl-1,3,5-

triazine / Monomer Reactivity Ratio / Glass Transition Temperature /

Dynamic Coefficient of Friction /
\end{abstract}

Polydimethylsiloxane is extremely interesting material having excellent properties, for example, water-repellency, easy peeling, lubricity, resistance to heat, resistance to weather, and high oxygen permeability. ${ }^{1-3}$ Particularly, block and/or graft copolymers containing polydimethylsiloxane have functional properties of both polydimethylsiloxane and another polymers. ${ }^{4-6}$ Graft copolymers consisting of macromonomers are very good materials because graft copolymers show the independent behavior of the backbone part and the graft part.

Works on graft copolymers using polydimethylsiloxane macromonomers were reported. Kawakami ${ }^{7,8}$ reported the preparation of siloxane graft copolymers and the effective surface modification of PMMA films by adding small amounts of siloxane graft copolymers. Cameron ${ }^{9,10}$ reported the monomer reactivity ratio of siloxane macromonomers towards styrene and acrylonitrile. Tsukahara ${ }^{11}$ reported the effects of thermodynamic repulsive interactions between the polymer reaction species on copolymerization behavior of polydimethylsiloxane macromonomers with methyl methacrylate.

Polymers with amino-s-triazine rings are hydrophilic, rigidly, and heat-stable (high glass transition temperature) ones. ${ }^{12-14}$

In this work the radical copolymerization of polydimethylsiloxane macromonomers with two isopropenyl-1,3,5-triazines, 2-amino-4anilino and 4-( $N$-octadecylanilino)-6-isopropenyl-1,3,5-triazine (AAIT and N18), was investigated. $T_{\mathrm{g}}$ and the coating surface of poly(triazines-graft-silicone) containing both the rigid triazine and the flexible siloxane grafts are reported. 
<smiles>C=C(C)c1nc(N)nc(Nc2ccccc2)n1</smiles>

AAIT<smiles>[10BH2]C([18OH])N(c1ccccc1)c1nc(N)nc(C(C)C)n1</smiles>

N18

Scheme 1. Structures of isopropenyl triazines.

\section{EXPERIMENTAL}

\section{Materials}

Tetrahydrofuran (THF) and dimethyl sulfoxide (DMSO) were purified by the ordinary method and distilled over calcium hydride under nitrogen.

2-Amino-4-anilino-6-isopropenyl-1,3,5-triazine $^{13}$ (AAIT) and 2-Amino-4-( $N$-octadecylanilino)-6-isopropenyl-1,3,5-triazine ${ }^{14}$ (N18) were prepared as described in the previous paper. AAIT: yields $87.5 \%, \mathrm{mp} 175^{\circ} \mathrm{C}$. Elemental analysis. Found: C, 64.01\%; H, $5.74 \% \dagger \mathrm{N}, 30.77 \%$. Calcd: C, 63.42\%, H, $5.77 \% ; \mathrm{N}, 30.81 \%$ for $\mathrm{C}_{12} \mathrm{H}_{13} \mathrm{~N}_{5}$. N18: yields $74.9 \%, \mathrm{mp} 75^{\circ} \mathrm{C}$. Elemental analysis. Found: C,75.45\%, H, 9.93\%, N, 14.68\%. Calcd: C, $75.11 \% ; \mathrm{H}, 10.29 \% ; \mathrm{N}, 14.59 \%$ for $\mathrm{C}_{30} \mathrm{H}_{49} \mathrm{~N}_{5}$.

\section{Measurements}

Molecular weights of polymers were determined by gel permeation chromatography (GPC) on a TOSOH HLC-803D with G2000H, $\mathrm{G} 3000 \mathrm{H}$, and $\mathrm{G} 5000 \mathrm{H}$ TSK gel-columns and/or on a TOSOH HSC-802UR with G3000H8, G2000H8 TSK gel-columns in a differential refractometric detector in THF. The column was calibrated with monodisperse polystyrene standard samples. ${ }^{1} \mathrm{H}$ NMR spectra were obtained in $\mathrm{CDCl}_{3}$ with a $200 \mathrm{MHz}$ Varian XL-200 instrument.

$T_{\mathrm{g}}$ was determined by differential scanning calorimetry (DSC) on a Rigaku-Denki DSC8230 at a heating rate of $20^{\circ} \mathrm{C} \mathrm{min}^{-1}$.

Dynamic viscoelasticity was measured with a viscoelastic spectrometer VES-F-III (Iwa- moto Seisakusho Co., Ltd.). The frequency and heating rate were $10 \mathrm{~Hz}$ and $2.5^{\circ} \mathrm{Cmin}^{-1}$, respectively.

Dynamic coefficient of friction of coating surface was measured with a surface property tester HEIDON-14 (Shinto Scientific Co., Ltd.) at $23 \pm 2{ }^{\circ} \mathrm{C}$ and $50 \% \mathrm{RH}$. The contact head was a stainless sphere $(10 \mathrm{~mm}$ diameter $)$ and crosshead speed was $150 \mathrm{~mm} \mathrm{min.}^{-1}$

\section{Macromonomers}

Methacrylate-terminated polydimethylsiloxane macromonomers (MAS) were supplied by Toshiba Silicone Co., Ltd. and Chisso Co., Ltd. Their structures and characterization are shown in Table I. MAS2 was purified by distillation under reduced pressure. MAS15, MAS16, MAS45, MAS68, and MAS133 were purified by washing with a large quantity of methanol and vacuum drying to constant weight. The molecular weights of macromonomers were determined by GPC and ${ }^{1} \mathrm{H}$ NMR and formed essentially the same. Macromonomers may thus have about one methacrylate end group per one molecule.

\section{Copolymerization}

Polydimethylsiloxane macromonomer was copolymerized with isopropenyl-1,3,5-triazine (AAIT or N18) in THF solution with AIBN as an initiator at $60^{\circ} \mathrm{C}$. All reaction solutions were homogeneous and transparent in polymerization. The graft copolymer was precipitated in excess $n$-hexane and dried in vacuo. Poly(AAIT-graft-MAS68 or MAS133) containing below $40 \mathrm{wt} \%$ of AAIT content, and 
Table I. Characterization of silicone macromonomers $\left(\mathrm{MAS}_{n+1}\right)$

\begin{tabular}{|c|c|c|c|c|c|c|}
\hline \multirow{3}{*}{ Macromonomer } & \multicolumn{4}{|c|}{ 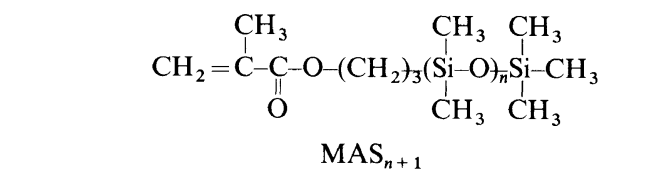 } & \multirow{3}{*}{$\frac{{ }^{1} \mathrm{H} \text { NMR }}{\bar{M}_{n}}$} & \multirow{3}{*}{$f^{\mathrm{b}}$} \\
\hline & \multicolumn{4}{|c|}{$\mathrm{GPC}^{\mathrm{a}}$} & & \\
\hline & $n$ & $\bar{M}_{n}$ & $\bar{M}_{w}$ & $\bar{M}_{w} / \bar{M}_{n}$ & & \\
\hline MAS2 & 1 & - & - & - & 268 & - \\
\hline MAS15 & 14.4 & 1270 & 1430 & 1.12 & 1230 & 1.0 \\
\hline MAS16 & 15.2 & 1330 & 1540 & 1.16 & 1180 & 1.1 \\
\hline MAS45 & 43.6 & 3430 & 4010 & 1.17 & 3660 & 0.9 \\
\hline MAS68 & 67.2 & 5180 & 5870 & 1.13 & 5760 & 0.9 \\
\hline MAS133 & 131.8 & 9970 & 11400 & 1.14 & 10700 & 0.9 \\
\hline
\end{tabular}

a Molecular weight was calculated by using calibration curve of polystyrene standard samples.

${ }^{\mathrm{b}}$ End functionality was calculated from $f=\bar{M}_{n}(\mathrm{GPC}) / \bar{M}_{n}\left({ }^{1} \mathrm{H}\right.$ NMR $)$.

poly(N18-graft-MAS68 or MAS133) could not be purified because of similarity of solubility of both macromonomers and graft copolymer.

\section{RESULTS AND DISCUSSION}

\section{Copolymerization Behavior of Macromonomers}

The relationship between the composition of the monomer feeds and that of the graft copolymers, poly(AAIT-graft-MAS), is shown in Figure 1. The monomer reactivity ratios $\left(r_{1}\right.$, $r_{2}$ ) obtained from the Kelen-Tüdös plots are shown in Table II. $r_{1}$ decreased from 0.50 to 0.10 and $r_{2}$ was increased from 1.97 to 6.47 with the molecular weights of the macromonomers. These phenomena are in agreement with the copolymerization with silicone macromonomers and styrene or acrylonitrile. ${ }^{9,10}$ The reason of the relative lowering of the reactivity of the macromonomers may be lowering of the compatibility between silicone macromonomers and AAIT with of molecular weight of the macromonomers. The results of the copolymerization of silicone macromonomers and N18 are shown in Figure 2 and Table II. The variation of $r_{1}$ and $r_{2}$ was similar to the case of copolymerization with AAIT. $r_{1}$ decreased from 0.58 to 0.30 and $r_{2}$ increased

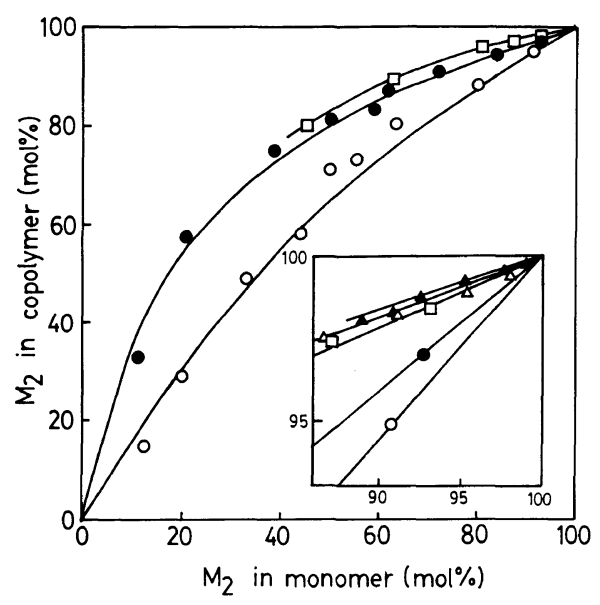

Figure 1. Monomer-copolymer composition curves for the copolymerization of AAIT $\left(\mathrm{M}_{2}\right)$ with MAS2 $(\mathrm{O})$,

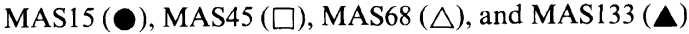
in THF.

from 0.49 to 1.09 with the molecular weights of the macromonomers. The value of $1 / r_{2}$, i.e., the relative reactivity of macromonomers, decreased with increase of the molecular weights. By comparison with AAIT and N18, the relative reactivity of AAIT was fairly higher than that of N18.

The solubilities of silicone macromonomer, poly(AAIT), and poly(N18) were tested. 
Table II. Monomer reactivity ratios for the copolymerization of isopropenyl triazines with silicone macromonomers ${ }^{\mathrm{a}}$

\begin{tabular}{cllll}
\hline $\mathrm{M}_{2}$ & \multicolumn{1}{c}{$\mathrm{M}_{1}$} & $r_{1}{ }^{\mathrm{b}}$ & $r_{2}{ }^{\mathrm{b}}$ & $1 / r_{2}$ \\
\hline \multirow{2}{*}{ AAIT } & BuMA $^{\mathrm{c}}$ & 0.45 & 1.27 & 0.79 \\
& MAS2 & 0.50 & 1.97 & 0.51 \\
& MAS15 & 0.19 & 3.94 & 0.25 \\
& MAS45 & 0.14 & 4.90 & 0.20 \\
& MAS68 & 0.10 & 6.03 & 0.17 \\
\multirow{2}{*}{ 18 } & MAS133 $^{3}$ & 0.10 & 6.47 & 0.15 \\
& BuMA $^{\mathrm{c}}$ & 0.58 & 0.40 & 2.50 \\
& MAS2 & 0.62 & 0.42 & 2.38 \\
& MAS15 & 0.30 & 1.09 & 0.92 \\
& MAS45 & 0.04 & 1.08 & 0.93 \\
\hline
\end{tabular}

a Copolymerizations was carried out in THF.

b $r_{1}$ and $r_{2}$ were calculated from the Kelen-Tüdös plots.

c $n$-Butyl methacrylate.

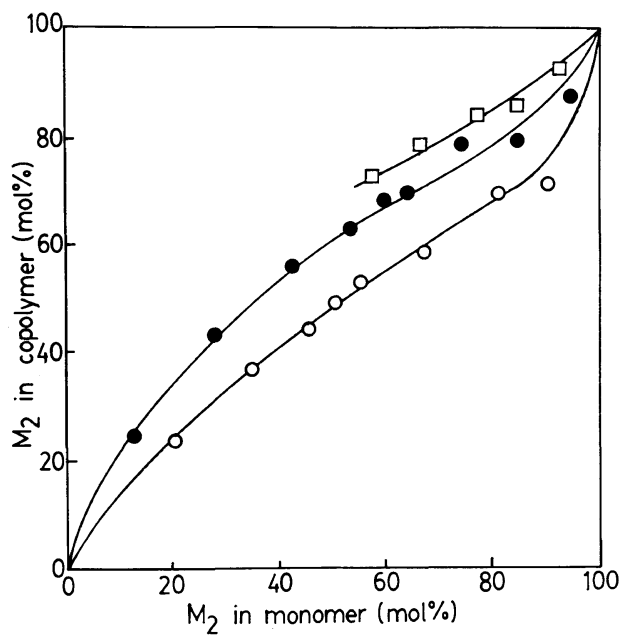

Figure 2. Monomer-copolymer composition curves for the copolymerization of $\mathrm{N} 18\left(\mathrm{M}_{2}\right)$ with MAS2 (O), MAS16 (-), and MAS45 ( $\square$ ) in THF.

Silicone macromonomer was soluble in nonpolar solvent such as hexane, benzene, and dioxane, and weak polar solvents such as ether, chloroform, pyridine, and THF, whereas insoluble in dipolar aprotic solvents. Poly(AAIT) was insoluble in non-polar solvents, and soluble in THF and dipolar aprotic solvents. Poly(N18) was well-soluble in weak polar and non-polar solvent rather than in dipolar aprotic solvents. The solubility of poly(N18) was similar that of silicone macromonomers. A common good solvents for both poly(N18) and silicone macromonomers were THF, chloroform, toluene, and benzene. These results suggest that the decrease of relative reactivity of macromonomers with the increase of the molecular weights is due to differences of solubility of between graft copolymer radicals and macromonomers.

\section{Thermal Behavior}

$T_{\mathrm{g}}$ of the poly(AAIT-graft-MAS) and poly(AAIT-co-BuMA) is plotted in Figure 3 against AAIT content of the graft copolymers. $n$-Butyl methacrylate (BuMA) was used as a model compound. $T_{\mathrm{g}}$ of the poly(AAIT-coBuMA) was observed on a straight line between $T_{\mathrm{g}} \mathrm{s}$ of poly(AAIT) and poly(BuMA). $T_{\mathrm{g}}$ of poly(AAIT-graft-MAS2) deceased on the straight line above $20 \mathrm{wt} \%$ of AAIT content region and the rapidly decrease in $T_{\mathrm{g}}$ was observed below $20 \mathrm{wt} \%$. $T_{\mathrm{g}}$ of the poly(AAITgraft-MAS 15 or MAS16) slightly decreased above $40 \mathrm{wt} \%$ of the AAIT content region and rapidly decreased under $40 \mathrm{wt} \%$ of the AAIT content region and rapidly decreased under $40 \mathrm{wt} \%$. $T_{\mathrm{g}}$ above $40 \mathrm{wt} \%$ of AAIT was higher than that of poly(AAIT-graft-MAS2 or BuMA). Further, the second $T_{\mathrm{g}}$ peak was observed at about $-120^{\circ} \mathrm{C}$ with rapid decrease of the first $T_{\mathrm{g}}$. This suggests that poly(AAITgraft-MAS15 or MAS16) has a phase separation structure. Above $40 \mathrm{wt} \%$ of the AAIT content region, continuous phase was poly(AAIT) and dispersed phase was poly(MAS15 or MAS16). Below $40 \mathrm{wt} \%$ of AAIT content region, phase inversion occurred. The continuous phase was poly(MAS15 or MAS16) and dispersed phase was poly(AAIT).

$T_{\mathrm{g}}$ of poly(AAIT-graft-MAS68 or MAS133) containing above $50 \mathrm{wt} \%$ of AAIT content region was similar to $T_{\mathrm{g}}$ of poly(AAIT). This indicates that phase separation further proceeded with increase of molecular weights of the macromonomers. The reason for the phase 


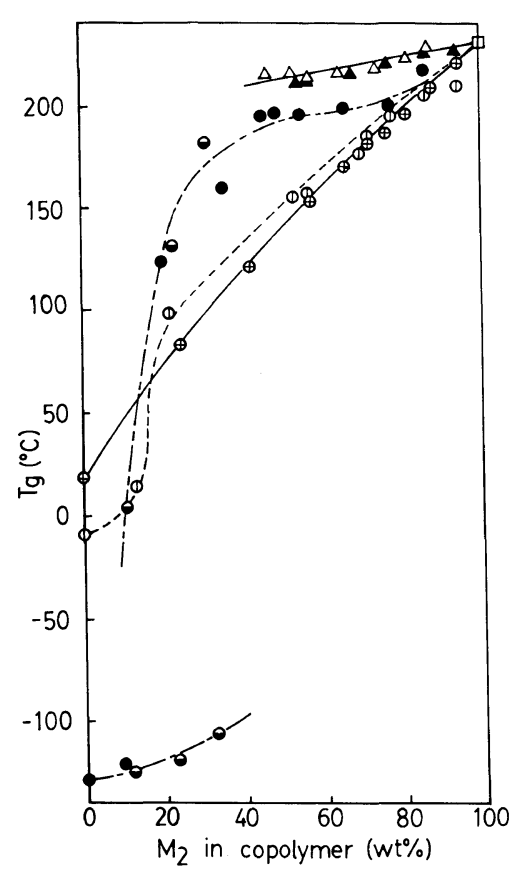

Figure 3. Relationship between $T_{\mathrm{g}}$ and AAIT content in copolymer of AAIT $\left(\mathrm{M}_{2}, \square\right)$ with butyl methacrylate $(\oplus)$, $\operatorname{MAS} 2(\oplus), \operatorname{MAS} 15(\bigcirc), \operatorname{MAS} 16(\ominus), \operatorname{MAS} 68(\triangle)$, and $\operatorname{MAS133}(\mathbf{\Delta})$.

separation may be that the compatibility between poly(AAIT) and silicone additionally decrease with increase of molecular weights of macromonomers.

Figure 4 shows the dynamic viscoelastic properties of the poly(AAIT-graft-MAS16) containing $22 \mathrm{wt} \%$ of AAIT. From the storage modulus $\left(E^{\prime}\right)$, loss modulus $\left(E^{\prime \prime}\right)$, and tangent delta $(\operatorname{Tan} \delta)$, poly(AAIT-graft-MAS16) have $T_{\mathrm{g}}$ about $-100^{\circ} \mathrm{C}$, which is in accordance with DSC measurements.

The relation of $T_{\mathrm{g}}$ and N18 content of poly(N18-graft-MAS) and poly(N18-coBuMA) is plotted in Figure 5. $T_{\mathrm{g}}$ of the copolymer of N18 with BuMA or MAS2 was observed on the straight line between $T_{\mathrm{g}}$ of the homopolymers. This suggests that these polymers are homogenous. $T_{\mathrm{g}}$ of poly(N18graft-MAS16)s little decreased above $60 \mathrm{wt} \%$ of the N18 content region and decreased with decrease of $\mathrm{N} 18$ content region below $60 \mathrm{wt} \%$.

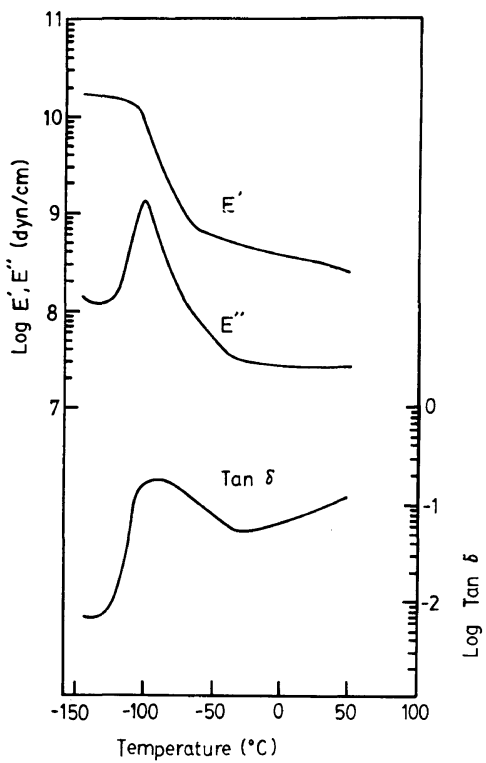

Figure 4. Temperature dependence of the storange modulus $\left(E^{\prime}\right)$, the loss modulus $\left(E^{\prime \prime}\right)$, and tengent delta (Tan $\delta$ ) for poly(AAIT-graft-MAS16) containing $22 \mathrm{wt} \%$ of AAIT.

The second $T_{\mathrm{g}}$ of about $-120^{\circ} \mathrm{C}$ was not observed with increase of MAS16 content. This suggests that phase separation occurred above $60 \mathrm{wt} \%$ of N18 contents. The phase would invert to composite included structure below $60 \mathrm{wt} \%$ of $\mathrm{N} 18$ contents region.

It seems that the difference of $T_{\mathrm{g}}$ variation with AAIT and N18 copolymers arose from micro-phase structures of graft copolymers. Thus, copolymers containing N18 having $\mathrm{N}$-octadecyl substituent showed increase of hydrophobic properties. The structures of copolymers were not show micro-phase separation and poly(N18) part was compatible with the silicone part.

\section{Dynamic Coefficient of Friction}

To estimate the coating surface of graft copolymer containing silicone, the dynamic coefficient of friction (DCF) of coating surface was measured. The value of DCF versus the number average molecular weight of copolymerized silicone macromonomers is plotted 


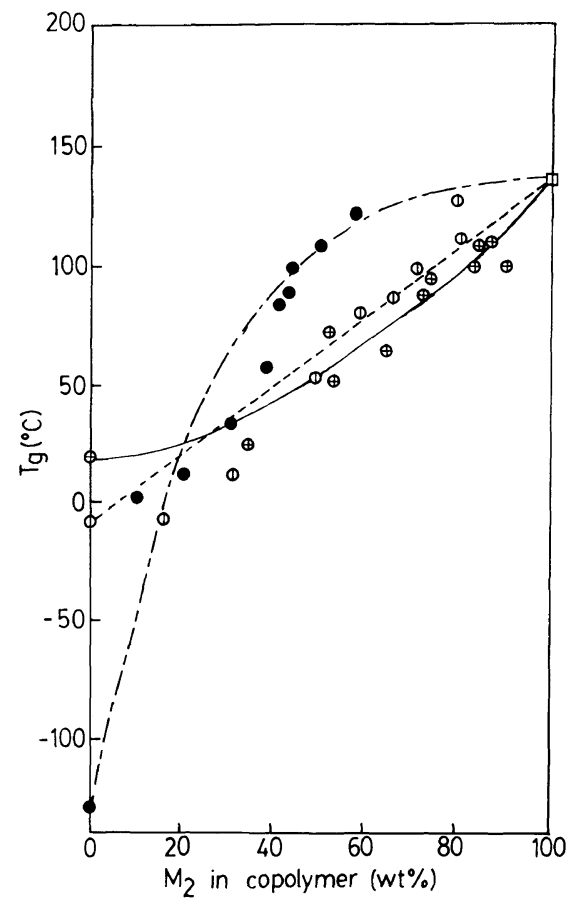

Figure 5. Relationship between $T_{\mathrm{g}}$ and N18 content in copolymer of $\mathrm{N} 18\left(\mathrm{M}_{2}, \square\right)$ with butyl methacrylate $(\oplus)$, MAS2 (๑), and MAS16 (O).

in Figures 6 and 7. DCF decreased with increase of silicone content and the molecular weights of macromonomers. The variation of DCF of poly(AAIT-graft-MAS)s is shown in Figure 6. DCF of the poly(AAIT-graftMAS) for $20 \mathrm{wt} \%$ of silicone macromonomer was nearly constant below about 5000 of $M_{n}$ (MAS68). Above $40 \mathrm{wt} \%$ of macromonomers, DCF decreased with increase of molecular weights of macromonomers. DCF of poly(AAIT-graft-MAS) containing $60 \mathrm{wt} \%$ macromonomers rapidly decreased compared with that of the small molecular weight macromonomer (MAS16).

The variation of DCF of poly(N18-graftMAS)s is shown in Figure 7. By comparing with poly(AAIT-graft-MAS), the DCF of the poly(N18-graft-MAS) was rapidly decreased by introducing a small amount $(20 \mathrm{wt} \%)$ of macromonomers (MAS16) with relatively short silicone chains.

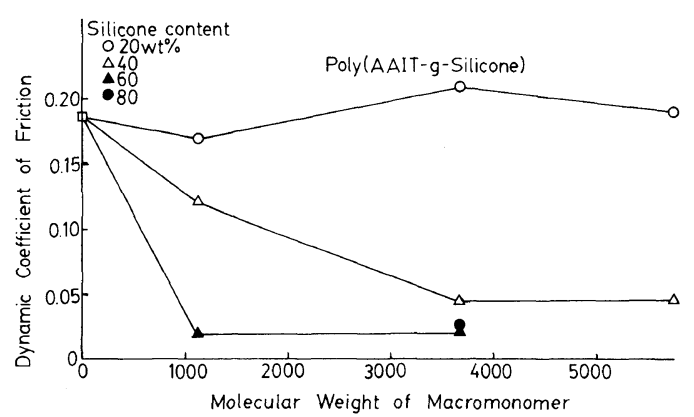

Figure 6. Dynamic coefficient of friction of poly(AAITgraft-MAS) versus the number average molecular weight of silicone macromonomers: silicone contents of $20 \mathrm{wt} \%$ $(\bigcirc), 40 \mathrm{wt} \%(\triangle), 60 \mathrm{wt} \%(\mathbf{\Delta})$, and $80 \mathrm{wt} \%$

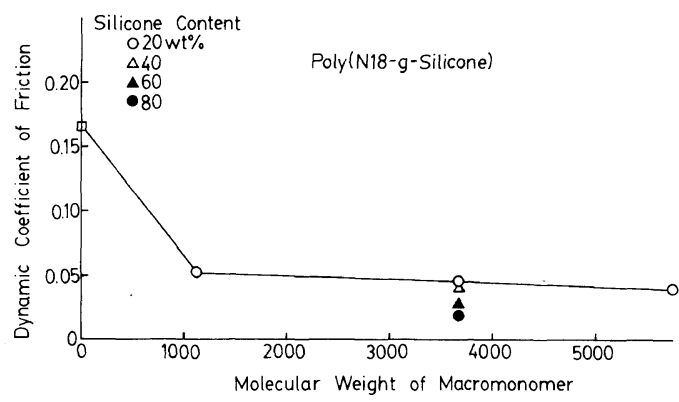

Figure 7. Dynamic coefficient of friction of poly(N18graft-MAS) versus the number average molecular weight of silicone macromonomers: silicone contents of $20 \mathrm{wt} \%$ (○), $40 \mathrm{wt} \%(\triangle), 60 \mathrm{wt} \%(\mathbf{\Delta})$, and $80 \mathrm{wt} \%(\bullet)$.

These results indicate that the variation of the coating surface occurs not only by the difference of molecular weights of silicone macromonomers but also by the difference of isopropenyl triazines.

The variation of DCF with the difference of isopropenyl triazines would be attributable to the difference of microstructures of two kinds of graft copolymers. That is, isopropenyl triazines become hydrophobic by the introduction of $N$-octadecyl substituent and had good compatibility to silicone macromonomers. Thus, silicone is dispersed homogeneously in coating films and rapid decrease of the DCF occurs on introducing small amounts of silicone macromonomers. 


\section{REFERENCES}

1. K. Maeda and K. Azuma, Kobunshi Kakou, 40, 182 (1991).

2. H. Inoue, A. Matsumoto, K. Matsukawa, A. Ueda, and S. Nagai, J. Appl. Polym. Sci., 40, 1917 (1990).

3. L. M. Clements, T. Hsu, S. S. Kantner, M. Mazurek, and T. Shibano, Setchaku, 32, 227 (1988).

4. B. Wang and S. Krause, J. Polym. Sci., Polm. Phys. Ed., B26, 2237 (1988).

5. H. Inoue, A. Ueda, and S. Nagai, J. Appl. Polym. Sci., 35, 2039 (1988).

6. D. Feng, G. L. Wilkes, and J. V. Crivello, Polymer, 30, 1800 (1989).

7. Y. Kawakami, R. A. H. Murthy, and Y. Yamashita,
Makromol. Chem., 185, 9 (1984).

8. Y. Kawakami, T. Aoki, Y. Yamashita, M. Hirose, and A. Ishitani, Macromolecules, 18, 580 (1985).

9. G. G. Cameron and M. S. Chiholm, Polymer, 26, 437 (1985).

10. G. G. Cameron and M. S. Chiholm, Polymer, 27, 1420 (1986).

11. Y. Tsukahara, N. Hayashi, X. L. Jiang, and Y. Yamashita, Polym. J., 21, 377 (1989).

12. Y. Yuki, T. Kakurai, and T. Naguchi, Bull. Chem. Soc. Jpn., 43, 2123 (1970).

13. Y. Yuki and T. Oouchi, Kobunshi Ronbunshu, 31, 67 (1974).

14. H. Kunisada, Y. Yuki, S. Kondo, J. Miyatake, and C. Maeda, Polym. J., 22, 559 (1990). 